\title{
Influencia de los gastos de campaña en los resultados de las elecciones federales de 1997
}

J. Ramón Gil-García •

Rodrigo Sandoval Almazán *^

Introducción

Actualmente los partidos
políticos en todo el mundo
centran sus estrategias
político-electorales en los
consejos de múltiples y
costosos asesores de mercadotecnia política. Difícil-

Recientemente se ha incrementado la preocupación por el control de los gastos de campaña efectuados por los distintos partidos políticos. Se argumenta que los excesivos gastos provocan inequidad entre

las diferentes fuerzas políticas, ya que influyen fuertemente en la decisión de los ciudadanos a votar por la opción partidista que más recursos gaste durante las campañas. Asimismo se ha cuestionado el uso de recursos públicos para el financiamiento de estas campañas políticas. Este estudio analiza la incidencia de los gastos de campaña en los resultados electorales. La relación de los gastos de campaña con la votación emitida es estadísticamente significativa tanto para los partidos en su conjunto -lo que explica de alguna forma el abstencionismo - como para cada una de las fuerzas políticas por separado, mostrando de forma preliminar la eficiencia en el uso de estos gastos. Sin embargo, en ambos casos los coeficientes estimados muestran una débil relación causal.

Palabras clave: gastos, campañas, mercadotecnia política, elecciones, votación.

Espiral, Estudios sobre Estado y Sociedad mente un partido o un candidato escapan a esta tendencia mundial $y$ al uso de diferentes herramientas de mercadotecnia para apoyar sus campañas. Como consecuencia de este hecho, la mercadotecnia política utiliza una gran cantidad de instrumentos y técnicas para desempeñar su labor; dichas herramientas teóricas o prácticas implican un enorme gasto que los partidos y candidatos deben cubrir: los gastos de campaña.

- Profesor investigador en el Centro de Investigación y Docencia Económicas y profesor de la Facultad de Ciencias Políticas y Administración Pública, Universidad Autónoma del Estado de México. Estudiante de doctorado en el Rockefeller College of Public Affairs and Policy, University at Albanyt jgil-garcia@ctg.albany.edu

Estudiante doctoral del programa en Administración. Profesor de planta del Departamento de Humanidades, ITESM, campus Toluca, y director del Journal on Information and Poverty.

rodrigo.sandoval@itesm.mx 
En nuestro país los gastos de campaña o gastos electorales tienen una breve historia. A partir de las reformas de 1997 a la Constitución y con la creación del Cofipe se comenzó a hablar de los gastos electorales, los topes de campaña y el financiamiento público a los partidos políticos. Antes de ello, parecía haber poco interés o poca capacidad de influencia por parte de los partidos políticos en cuanto a temas relacionados con los recursos utilizados en las campañas políticas.

El objetivo de este estudio es analizar la influencia que los gastos de campaña tuvieron en la votación obtenida y en la reducción del abstencionismo durante las elecciones federales de 1997. Las elecciones intermedias normalmente presentan características que las distinguen de las elecciones concurrentes, entre las que destaca un nivel más alto de abstencionismo, debido tal vez a que la gente considera más relevante votar por el presidente o por su gobernador, que por sus legisladores.

Para lograr el objetivo establecido, este artículo se ha dividido en cuatro apartados. El primero establece la conceptualización y algunas características de la mercadotecnia política como instrumento por medio del cual se ejercen los gastos de campaña en la búsqueda del voto ciudadano. El segundo establece la forma en que se han estudiado los gastos de campaña y su relación con los procesos electorales. Un tercer apartado describe la metodología utilizada para este estudio, así como las principales fuentes de datos que se tuvieron como base para el análisis cuantitativo. En el cuarto apartado se llevan a cabo diversos análisis de regresión, así como tablas de correlación y algunas pruebas estadísticas para verificar los supuestos que permiten usar mínimos cuadrados ordinarios (MCO). Finalmente, se enumeran algunas conclusiones y reflexiones finales a la luz del marco teórico inicialmente expuesto.

Es importante mencionar que los resultados obtenidos en este trabajo, si bien no se pueden generalizar a todo tipo de 
Influencia de los gastos de campaña en los resultados de las elecciones

comicios, si muestran algunas lecciones que en cuanto a eficiencia del gasto y reducción del abstencionismo se tuvieron en una elección intermedia que comenzaba a ser altamente competida. De hecho, la siguiente elección federal que tuvo lugar en México dio como resultado el cambio del partido político en el poder, acabando con una época de cuasi democracia y falta de control ciudadano en los gastos que cada partido podía efectuar durante una contienda electoral.

Futuros estudios podrán concentrarse en el análisis comparativo de diferentes patrones de gasto en elecciones intermedias y concurrentes. Temas como el tratado en este artículo tienen gran relevancia en al menos dos debates públicos: a) hasta qué punto el financiamiento público a las campañas políticas tiene un impacto positivo en el número de ciudadanos que deciden votar en una elección, y b) de qué forma los gastos electorales en distintos rubros influyen la obtención de votos por las distintas fuerzas políticas.

\section{Mercadotecnia política y gastos de campaña}

Esta sección está dedicada a la exposición de algunos de los postulados fundamentales de la mercadotecnia política y su relación con los gastos que se llevan a cabo en las campañas electorales. Para tener un mejor entendimiento de la relación entre los gastos de campaña y los resultados electorales es necesario entender lo que es la mercadotecnia y ubicar en este contexto a la mercadotecnia política.

Desde el punto de vista estrictamente político, el concepto de mercadotecnia implica que las necesidades primarias de los consumidores son la principal preocupación de los candidatos y que éstos deben identificar y tratar de resolver tales necesidades. Desde el punto de vista comercial, la mercadotecnia está basada en la premisa de que todas las compañías deben planear sus operaciones orientadas al consumidor para elevar el nivel de ventas (O’Cass, 1996). 
El concepto de marketing ${ }^{1}$ más aceptado es el que sostiene la American Marketing Association:

Marketing es el proceso de planificación y ejecución del concepto, precio, promoción y distribución de ideas, bienes y servicios para crear intercambios que satisfagan los objetivos del individuo y de la organización (Luque, 1997).

Cuando un partido o candidato aplica este concepto al proceso político, debe estar en posición de adaptar su plataforma con la finalidad de satisfacer a los votantes potenciales.

La mercadotecnia es entonces el conjunto de actividades, tanto de individuos como de organizaciones, que se encaminan a facilitar y estimular intercambios dentro de un grupo de fuerzas externas dinámicas, para conseguir un fin. La tarea del marketing inicia con el proceso de detección y descubrimiento de las necesidades presentes y latentes de los consumidores y sus perspectivas futuras anticipando la evolución de las mismas (Braidot, 1993: 7).

El francés Philippe Maarek (1997) establece que el marketing político puede definirse como: "una verdadera política de comunicación política, una estrategia global que comprende el diseño, la racionalización y la transmisión de la comunicación política”. El concepto de Herreros (1989) presenta la distinción entre mercadotecnia política y mercadotecnia electoral:

El marketing político, en general, debe entenderse como el conjunto de técnicas empleadas para influir en las actitudes y en las conductas ciudadanas a favor de ideas, programas y actuaciones de organismos o personas determinadas que detentan el poder, intentan mantenerlo y consolidarlo, o aspiran a conseguirlo. El marketing electoral se refiere

I. La palabra marketing significa mercadotecnia y ambos vocablos se utilizarán indistintamente en el cuerpo de este artículo. 
Influencia de los gastos de campaña en los resultados de las elecciones

con exclusividad al planteamiento, realización y difusión de unos determinados mensajes, con ocasión de la puesta en marcha de procesos electorales, para designar el gobierno de una determinada comunidad política; se trata, por tanto, de una variante específica de marketing político.

Bajo la óptica de estos conceptos, veamos otros elementos que apoyan esta disciplina. Por ejemplo, la publicidad electoral ha ido cobrando gran fuerza en las últimas décadas. Ello se puede observar en lo que Naghi (1986: 168) comenta: "En 1846 Abraham Lincoln gastó menos de un dólar estadounidense para ser electo; en 1972 Richard Nixon gastó 60 millones de dólares durante tres meses para ser electo". En la actualidad estas cifras se han multiplicado de forma impresionante no sólo en Estados Unidos, sino en todos los países del mundo que viven un régimen democrático.

Otro concepto que requeriremos para el análisis es el perfil del electorado, que no es otra cosa que un conjunto de diversas variables de tipo demográfico, socioeconómico, condiciones actuales de vida del individuo, de actitudes políticas ante el partido y el candidato, etc. Según se definan las variables que influyan en el perfil, habrá diferentes perfiles en el mercado electoral.

Es recomendable conocer el comportamiento de los electores y desarrollar un modelo de comportamiento, con el fin de pronosticar con cierta confiabilidad por quién se inclinará la votación. Hay tres factores que influyen directamente en la actitud política del individuo: el temperamento político, las preocupaciones políticas y socioeconómicas del elector, y la personalidad de los candidatos. Los gastos electorales pueden ayudar a mejorar o fortalecer diferentes aspectos que de alguna forma favorezcan al candidato en cuestión.

Para finalizar este primer acercamiento, expondremos algunas de las características de los medios de comunicación más utilizados en las campañas electorales. La propaganda 
política utiliza todos los medios publicitarios posibles, incluyendo: Internet, radio, televisión, periódicos y revistas. La televisión ha traído un cambio sustancial en las campañas electorales, ya que la publicidad a través de este medio es muy poderosa, especialmente para los candidatos fotogénicos. Además, este tipo de propaganda llega hasta los hogares de gente de todos los niveles sociales, propiciando el acercamiento del candidato de igual manera a la gente más pobre que a los grupos económicamente más poderosos del país.

El alcance y la frecuencia de la estrategia de los medios publicitarios son los aspectos más importantes para el candidato. Por eso la televisión es el medio propagandístico preferido, pues su alcance a grandes masas y su repetición constante lo convierten en el instrumento ideal para sus campañas. Las investigaciones científicas realizadas en cuanto a la eficacia de este medio muestran que la audiencia de las campañas televisadas es muy elevada y que su influencia sobre los electores es notable. Es un medio privilegiado para acrecentar la notoriedad de un candidato inicialmente poco conocido.

Se ha comprobado que, ante la pantalla, los electores son más susceptibles al espectáculo que a los discursos, y son más sensibles a los personajes que a las ideas. Por tanto, se puede concluir que si se puede utilizar la televisión, resulta el soporte privilegiado de una campaña personalizada. En contraparte, la radio se utiliza como complemento de la campaña por televisión, es menos eficaz en las personas que no pueden seguir la campaña televisada y ayuda a los candidatos a dar a conocer sus ideas y planes de actividad política. Estos dos medios, en su conjunto, son tan relevantes que incluso en los reportes de los gastos de campaña del IFE, los partidos deben incluir sus gastos en estos dos conceptos en un apartado diferente a los otros gastos. 
Influencia de los gastos de campaña en los resultados de las elecciones

Otro elemento importante es el cartel, que contiene información visual breve y por lo general se fija en un muro. Un cartel bueno es aquel que emplea una ilustración visual grande para captar y mantener la atención, de modo que el transeúnte lea el mensaje escrito con pocas palabras, muy breve, pero altamente comprensible y persuasivo.

Las reuniones y el contacto personal de los candidatos en diversos sitios de reunión de cada ciudad o en las universidades, son una forma de comunicación masiva que sirve como propaganda electoral para generar cierta imagen del candidato. Debe recordarse que la personalidad, presentación e influencia personal ejercen un papel importante en la campaña electoral. El contacto personal del candidato con los líderes de opinión permite al candidato y a sus representantes desarrollar temas específicos.

Una estrategia un tanto diferente es utilizar el concepto de propaganda en un sentido general e integrador, pero dirigido a la comunicación política. En condiciones normales, el mensaje que se quiere propagar se transmite como mejor se puede; pero cuando se utilizan herramientas como la retórica, la publicidad o la técnica, el efecto y la magnitud de lo transmitido crece y se vuelve más eficaz.

Tanto la publicidad comercial como la propaganda política utilizan varios instrumentos que las dirigen a su objetivo central: vender un objeto o una idea. Estos instrumentos psicosociales (según la clasificación y definiciones de Roberto Fabregat) son: la conquista del espacio urbano, la prensa, el teatro y el cine, la radio, las técnicas de sonido y la televisión, y la celebración política.

El objetivo dentro de la propaganda política en la conquista del espacio urbano es atraer e impresionar por medio de la atención y la memoria (misma meta que los publicistas comerciales). Dentro de las ciudades modernas, es sencillo atraer la atención; sólo basta con aumentar tamaños, reforzar contornos y simplificar diseños. También se 
simplifican imágenes, palabras o frases para que queden como "marcas".

La historia del presente, como se le ha llamado a la prensa, tiene dos grandes ventajas: es cotidiana y sorpresiva. Registra, ordena y dirige día tras día el acontecer social. Además es sistemática, puntual, microenciclopédica y anónima, y fuente tradicional de información. Para la propaganda ideológica, el cine es un instrumento formidable, a condición de que no parezca directamente realizado con ese objetivo.

La propaganda política trabaja específicamente con instintos e impulsos que asumen en forma rápida contornos colectivos: miedo y odio. El fin de la repetición constante es captar la atención del individuo, y bajo la premisa de que un mensaje es más fácil de asimilar si se repite muchas veces, el siguiente paso consiste en reiterar la sensación para que quede en la memoria; por último el individuo debe considerar el mensaje que debe afectar su opinión y le dé importancia, por lo tanto el mensaje más repetido será el más trascendental.

En suma, la mercadotecnia política utiliza los medios de comunicación, la persuasión, la comunicación política y la propaganda política para llevar su mensaje a los electores y convencerlos de que su "producto-candidato" es la mejor opción que tienen y por lo tanto debe ser electo. Para todas estas acciones los diferentes partidos y candidatos utilizan recursos públicos y contribuciones de diferentes organizaciones e individuos. Pareciera ser que, de forma similar a la mercadotecnia comercial, el partido que más y mejor gaste en una eficaz campaña publicitaria será el que tenga las mayores posibilidades de obtener el triunfo en los comicios.

\section{Gastos de campaña y resultados electorales}

Sin lugar a dudas los partidos políticos tienen una gran cantidad de actividades previas a la elección y durante el pro- 
Influencia de los gastos de campaña en los resultados de las elecciones

ceso electoral. No está por demás mencionar algunas de estas acciones: diseñan un programa o plataforma política que proponen a los electores, seleccionan sus candidatos, adiestran a sus miembros, diseñan su sistema de comunicación electoral, establecen sus tácticas y estrategias contra sus adversarios, etc. Como ya mencionamos, todo ello implica el uso de medios y recursos; a dichos recursos se les denomina gastos de campaña o financiamiento a los partidos políticos.

Las investigaciones en ésta área han generado una teoría electoral del gasto. Payne (1991) establece algunos postulados de esta teoría: 1. La idea de que los votantes están reflejando su propio interés racional votando por los representantes que gastan más. 2. Los votantes están mal informados y son irracionales, dejando que las elecciones sean dominadas por los "votantes de especial interés". 3. Las donaciones y los gastos de campaña son la clave del éxito.

Desde la óptica de Payne (1991), surgen también dos hipótesis sobre la influencia de los gastos de campaña en el proceso electoral:

a) La hipótesis de consecuencias electorales: incorpora la idea de que los representantes que gastan más obtienen beneficios electorales por ello.

b) Hipótesis de la inseguridad del legislador: la competición electoral y el "miedo al fracaso fuerzan a los representantes a aprobar más gasto del que, incluso ellos mismos, creen necesario.

Partiendo de la primera hipótesis, se supondría que a mayor gasto mayores probabilidades de triunfo, por que un mayor gasto permite una mejor identificación de las características del electorado; un mejor estudio sobre el contrincante posibilita una mejor elaboración de una plataforma política, favorece una mejor preparación, coordinación e incentivación de la organización en todo el proceso electoral mejorando inclusive la comunicación entre el candidato y el electorado.

Estado 8 No. 29 
Teodoro Luque (1996) analiza también el gasto electoral pero desde la óptica del marketing y lo divide en dos niveles:

a) El micro marketing, donde el gasto en las campañas es un problema de "fijación del presupuesto óptimo y de cómo repartirlo con eficacia".

b) El macro marketing, que incluye cuestiones como: 1. La limitación de la cuantía de gasto de las campañas políticas. 2. Las regulaciones del gasto, sus medios de obtención y su utilización 3. Subvenciones y ayudas a las campañas: financiación pública o privada. 4. Efecto que provoca la limitación del gasto sobre las distintas alternativas.

Continuando con el enfoque de marketing, también conviene analizar la percepción del consumidor o elector. Aunque no existen estudios realizados todavía sobre el tema del marketing político, en la mercadotecnia comercial Kirmani y Wright (1989) plantean una serie de explicaciones sobre cuándo y por qué el gasto de publicidad percibido influye en las expectativas de calidad para los consumidores, ambos autores establecen:

1. El gasto de publicidad percibido es un indicador del esfuerzo de marketing percibido y éste es un indicador de la confianza del empresario en la calidad de su producto.

2. Los consumidores perciben una correlación entre el gasto en publicidad y la calidad en algunos mercados.

3. El gasto percibido en publicidad es un signo de una fuerza financiera en la empresa.

Bajo estas afirmaciones de la mercadotecnia comercial, es importante preguntarse: ¿cómo se pueden trasladar estos argumentos al caso de la mercadotecnia política? Precisamente el objetivo del presente estudio es tratar de dar una respuesta a esta pregunta. En él tratamos de explorar la influencia de los gastos de campaña en la votación obtenida por los distintos partidos políticos, siguiendo de alguna forma algunas de las ideas plasmadas en el modelo 
Influencia de los gastos de campaña en los resultados de las elecciones

explicativo. Para Teodoro Luque (1997) existen tres modelos para analizar el gasto electoral:

1. El modelo explicativo, que se centra en la captación de la influencia de los gastos en el comportamiento de voto considerando una función de producción o según el concepto de éxito y la importancia de la imagen, interesándose en la conexión gastos y votos.

2. El modelo de contribución, que se centra en el porqué de las contribuciones a los gastos de campañas a partir del punto de vista del contribuyente que quiere maximizar su beneficio en la inversión de candidatos o asumiendo que la cuota de un candidato es la recompensa de su stock de capital político o el reconocimiento de su nombre.

3. Los modelos de asignación, donde su objetivo es la distribución óptima del presupuesto en una campaña electoral minimizando los costes de transmisión de información con relación a los otros competidores.

Como fue mencionado, bajo el enfoque de los modelos de análisis del gasto electoral de Luque, este trabajo se orienta fundamentalmente por premisas del primer modelo y busca explicar la relación entre gastos de campaña y votos obtenidos por los distintos partidos políticos en las elecciones federales de 1997.

\section{Metodología}

Esta sección tiene la finalidad de presentar la metodología utilizada para este estudio, así como las principales fuentes de datos consultadas. En este trabajo se utilizan regresión simple y múltiple como herramientas de análisis estadístico. Los resultados de esta técnica son complementados con tablas de correlación. La interpretación de los resultados derivados del análisis cuantitativo se lleva a cabo a la luz del marco teórico presentado en las primeras secciones de esta investigación. 


\section{Fuentes de datos}

La información de los votos válidos se obtuvo de los resultados del Instituto Federal Electoral (IFE) para los distintos partidos por distrito y un concentrado por estado, que fue lo que se utilizó para el análisis en este trabajo; pues se tomaron los resultados y los gastos que tuvieron los distintos partidos por entidad federativa. ${ }^{2}$

Los datos necesarios referentes a los gastos de campaña se obtuvieron de los informes de gastos de campaña que los distintos partidos políticos entregaron al Instituto Federal Electoral, con relación a las campañas para diputados federales en los distintos estados. ${ }^{3}$

\section{Modelos estadísticos}

Este trabajo desarrolla primordialmente dos modelos estadísticos. El primero se centra en entender la relación de los gastos totales de campaña y la votación total emitida. Como se puede observar, este modelo busca explicar si los gastos de campaña financiados en gran parte con recursos públicos ayudan a disminuir el problema del abstencionismo.

En este caso, el signo esperado entre los gastos de campaña y el total de la votación emitida es positivo, ya que se espera que a mayor gasto en una campaña electoral el porcentaje de personas que estén dispuestas a votar sea mayor. En este modelo tanto la votación como los gastos serán el total de los partidos políticos, ya que la finalidad es determinar qué tanto impactan los recursos en su conjunto en la votación total, y por tanto en la disminución del abstencionismo. La forma general del modelo es la siguiente: 
Influencia de los gastos de campaña en los resultados de las elecciones

\begin{tabular}{ll} 
& \multicolumn{1}{c}{ VOTVAL $=\beta_{1}+\beta_{2} \mathrm{PROP}+\beta_{3} \mathrm{OPE}+\beta_{4} \mathrm{TV}$} \\
Donde: & \\
VOTVAL $=$ & Votación válida de todos los partidos políticos. \\
$\mathrm{PROP}=$ & Gastos en propaganda de todos los partidos políticos. \\
$\mathrm{OPE}=$ & Gastos operativos de todos los partidos políticos. \\
$\mathrm{TV}=$ & Gastos en radio y televisión de todos los partidos políticos.
\end{tabular}

A pesar de que no existe la seguridad de que los partidos gastaron sus recursos de la forma indicada por el Instituto Federal Electoral, se entiende como gastos en propaganda todos aquellos gastos realizados en publicidad impresa, como carteles, folletos y espectaculares. Como gastos operativos se entiende el pago al equipo de campaña, a los asesores, la logística de los eventos, etc. Y los gastos en radio y televisión comprenden precisamente la publicidad llevada a cabo por estos medios.

El segundo modelo que se utilizará en este trabajo, intenta observar la influencia que tuvieron los gastos en propaganda, operativos y en radio y televisión, como variables exógenas, en la votación obtenida por cada uno de los partidos políticos que ganaron curules en las votaciones del 6 de julio de 1997, como variable endógena.

Se espera que la influencia de las tres variables sea positiva en la votación lograda por cada una de las organizaciones políticas. Por tanto, el parámetro del monto de los gastos en campaña en los tres rubros en los que se dividió deberá tener signo positivo respecto a la votación por cada uno de los partidos políticos. Como la idea es observar la eficiencia con la que los partidos utilizaron sus gastos de campaña, el modelo que se utilizará en principio para explicar la relación señalada es el siguiente:

$\operatorname{Ln}(\operatorname{VOTVAL})=\beta_{1}+\beta_{2} \operatorname{Ln}(\mathrm{PROP})+\beta_{3} \operatorname{Ln}(\mathrm{OPE})+\beta_{4} \operatorname{Ln}(\mathrm{TV})$

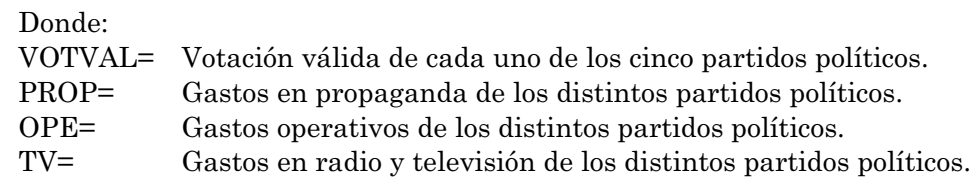


Gastos de campaña y votación total emitida: abstencionismo

Como se mencionó antes, se espera que el total de los gastos de campaña tenga un efecto positivo en el número de personas que deciden votar. Por tanto, los coeficientes de las diferentes variables explicativas se esperan positivos.

De los resultados obtenidos (tabla 1) se puede inferir que la variable que más influye, en la votación de los cinco partidos en su conjunto, es la propaganda en una relación de 11\% (es decir, ante un cambio en una unidad de gasto en propaganda, los votos crecen en 0.11 ) y una $t$ significativa. Los gastos operativos son los segundos en importancia con una relación de $6 \%$. Finalmente y de forma sorpresiva, los gastos en televisión y radio, además de tener un peso muy bajo en su asociación con el modelo, tiene una prueba $t$ no significativa, lo que nos dice que el parámetro para esta variable puede ser cero.

Tabla 1. Votación total emitida

\begin{tabular}{|c|c|c|}
\hline Variable & $\begin{array}{l}\text { Primer } \\
\text { modelo }\end{array}$ & $\begin{array}{l}\text { Segundo } \\
\text { modelo }\end{array}$ \\
\hline Constante & $\begin{array}{l}-48265.57 \\
(-0.944170)\end{array}$ & $\begin{array}{l}-155478.0 * * \\
(-3.161465)\end{array}$ \\
\hline Gastos en propaganda & $\begin{array}{l}0.119322^{* *} \\
(7.841458)\end{array}$ & \\
\hline Gastos operativos & $\begin{array}{l}0.063111^{* *} \\
(2.886956)\end{array}$ & \\
\hline Gastos en radio y televisión & $\begin{array}{l}0.011973 \\
(0.586839)\end{array}$ & \\
\hline Gastos totales & & $\begin{array}{l}0.063944^{* *} \\
(28.23303)\end{array}$ \\
\hline $\mathrm{R}^{2}$ & 0.975514 & 0.963729 \\
\hline $\mathrm{R}^{2}$ Ajustada & 0.972891 & 0.962520 \\
\hline F-Estadístico & $371.8426 * *$ & $797.1039 * *$ \\
\hline Prueba de White & $15.3256^{*}$ & $6.79 *$ \\
\hline
\end{tabular}

Las pruebas $t$ se encuentran en paréntesis bajo los coeficientes estimados. Los coeficientes con un asterisco $\left(^{*}\right)$ son significativos al nivel de $5 \%$, y los que tienen dos asteriscos $(* *)$ son significativos a $1 \%$. 
Influencia de los gastos de campaña en los resultados de las elecciones

Como los datos son de corte transversal, se pueden presentar principalmente dos problemas: multicolinealidad y hetorocedasticidad. Existen algunos síntomas que pueden indicarnos si nuestro modelo tiene presencia de multicolinealidad; entre ellos están: tener un $\mathrm{R}^{2}$ alto pero pocas razones $\mathrm{t}$ significativas, que existan altas correlaciones entre las parejas de regresores, un examen de las correlaciones parciales entre las variables explicativas, etcétera.

Sin embargo, ninguna de ellas nos dice de forma contundente si nuestro modelo presenta multicolinealidad o no. En este caso se espera que exista una alta correlación entre las variables independientes, ya que los líderes de los partidos pudieron haber usado una fórmula matemática como base inicial para la asignación de los gastos de campaña a los diferentes rubros.

Tabla 2. Matriz de correlación

\begin{tabular}{llll} 
Variables & PROP & OPE & TV \\
PROP & 1 & & \\
OPE & 0.8924 & 1 & \\
TV & 0.939416 & 0.945956 & 1 \\
\hline
\end{tabular}

La tabla 2 muestra una correlación alta entre las variables explicativas, pero no multicolinealidad perfecta. De hecho, puede demostrarse que aun con una muy alta multicolinealidad, los estimadores obtenidos mediante mínimos cuadrados ordinarios (MCO) seguirán siendo los mejores estimadores linealmente insesgados o MELI (Gujarati, 1997).

Por otro lado, con la finalidad de saber si el modelo presenta homocedasticidad, se realizó la prueba de White. El valor de esta prueba nos provee evidencia suficiente para afirmar que el modelo no presenta heterocedasticidad con $95 \%$ de confianza. De la misma forma se llevó a cabo la 
prueba de Breusch-Pagan-Godfrey, encontrándose evidencia de homocedasticidad a $99 \%$ de confianza.

Por otro lado, en el segundo modelo de abstencionismo, también presentado en la tabla 1 , se evitó el problema de multicolinealidad sumando los gastos para los tres diferentes rubros, dándoles la misma categoría en el modelo. El nuevo modelo quedó de la siguiente manera:

$$
\operatorname{VOTVAL}=\beta_{1}+\beta_{2} \mathrm{GTOCAMP}+\mu
$$

Donde vOTVAL representa la votación válida emitida por los cinco partidos políticos, y GTOCAMP se refiere a los gastos de campaña de los cinco partidos políticos, incluyendo propaganda, los operativos y los llevados a cabo en radio y televisión. Al ser una sola variable, eliminamos por ende el problema de multicolinealidad; y respecto a la heterocedasticidad; aplicando la prueba de White tenemos un $\mathrm{NR}^{2}$ $=6.79$, lo que parece indicar que a $95 \%$ de confianza no se tiene la presencia de heterocedasticidad. De nuevo se realizó la prueba de Breusch-Pagan-Godfrey, la cual sugiere que se tiene homocedasticidad a un nivel de $99 \%$ de confianza.

\section{Eficiencia de los gastos de campaña}

Como se mencionó en el apartado metodológico, el objetivo de esta sección no es únicamente analizar la influencia de los gastos de campaña en los resultados electorales, sino también explicar qué tipo de gasto es más eficiente para cada fuerza política.

La tabla 3 muestra los resultados del análisis de regresión múltiple para los cinco partidos políticos motivo de estudio. De los resultados se puede inferir la influencia de los distintos tipos de gasto de manera porcentual en la votación emitida por cada uno de los diferentes partidos políticos. Existe de igual forma una votación que no depende de las 
variables dentro del modelo, representada por la constante de la regresión, la cual se puede considerar cero para efectos prácticos.

Tabla 3. Log (votación recibida)

\begin{tabular}{|c|c|c|c|c|c|}
\hline Constante & $\begin{array}{l}-3.2691 \\
(-1.1489)\end{array}$ & $\begin{array}{l}-4.2503^{* *} \\
(-2.711)\end{array}$ & $\begin{array}{l}-1.0585^{*} \\
(-1.7197)\end{array}$ & $\begin{array}{l}-6.1806 * * \\
(-2.3845)\end{array}$ & $\begin{array}{l}-4.3245^{*} \\
(-2.1156)\end{array}$ \\
\hline $\begin{array}{l}\text { Log (gastos en } \\
\text { propaganda) }\end{array}$ & $\begin{array}{l}0.520356^{* *} \\
(2.3209)\end{array}$ & $\begin{array}{l}0.416942 * \\
(1.9042)\end{array}$ & $\begin{array}{l}0.3204 * * * \\
(3.7638)\end{array}$ & $\begin{array}{l}0.3847^{*} \\
(1.904)\end{array}$ & $\begin{array}{l}0.06611 \\
(0.60071)\end{array}$ \\
\hline $\begin{array}{l}\text { Log (gastos } \\
\text { operativos) }\end{array}$ & $\begin{array}{l}0.025770 \\
(0.116827)\end{array}$ & $\begin{array}{l}1.2078 * * * \\
(4.16502)\end{array}$ & $\begin{array}{l}0.2017 * * \\
(2.5265)\end{array}$ & $\begin{array}{l}0.2395 \\
(1.1161)\end{array}$ & $\begin{array}{l}0.095 \\
(0.4874)\end{array}$ \\
\hline $\begin{array}{l}\mathrm{Log} \text { (gastos } \\
\text { en radio y } \mathrm{TV} \text { ) }\end{array}$ & $\begin{array}{l}0.558835 \\
(1.679781)\end{array}$ & $\begin{array}{l}4.165022 \\
(-1.1606)\end{array}$ & $\begin{array}{l}0.4292 * * * \\
(4.3958)\end{array}$ & $\begin{array}{l}0.5393 * \\
(2.0289)\end{array}$ & $\begin{array}{l}0.9985^{* * * *} \\
(5.4948)\end{array}$ \\
\hline $\mathrm{R}^{2}$ & 0.6068 & 0.8494 & 0.9519 & 0.5819 & 0.8539 \\
\hline $\mathrm{R}^{2}$ ajustada & 0.5647 & 0.8333 & 0.9467 & 0.5371 & 0.8141 \\
\hline F-Estadístico & $14.4054^{* * * *}$ & $52.665^{* * * *}$ & $184.67 * * *$ & $12.99 * * *$ & $21.437 * *$ \\
\hline $\begin{array}{l}\text { Prueba de } \\
\text { White }\end{array}$ & $4.836^{* *}$ & $9.923 * *$ & $10.197^{* *}$ & $9.6592^{* *}$ & 9.6614 \\
\hline
\end{tabular}

Las pruebas $t$ se encuentran en paréntesis bajo los coeficientes estimados. Los coeficientes con un asterisco $\left(^{*}\right)$ son significativos al nivel de $10 \%$, los que tienen dos asteriscos $\left(^{* *}\right)$ son significativos a $5 \%$, y los señalados con tres asteriscos $(* * *)$ son significativos al nivel de $1 \%$.

Partido Acción Nacional (PAN)

En el caso del PAN, tanto los gastos en propaganda como los efectuados en radio y televisión son significativos a $97 \%$ y $88 \%$ de confianza, respectivamente. Se puede decir que ante un cambio en uno por ciento en los gastos en propaganda se logró un cambio en $0.52 \%$ en la votación obtenida; y que ante un incremento en uno por ciento en los gastos en televisión y radio se tuvo un aumento de $0.55 \%$ en los votos.

En el caso de los gastos operativos, además de que el parámetro es muy pequeño, apenas de 0.025 , su valor crítico 
$t$ es muy pequeño, lo que nos muestra que el parámetro no es significativo. La $\mathrm{R}^{2}$ ajustada igual a 0.5647 , muestra que existe un relativo buen ajuste al modelo en su conjunto.

Los problemas derivados de la multicolinealidad muy probablemente seguirán presentes pero, como ya lo mencionamos, no afectan de forma decisiva nuestros resultados, por lo que nos concentraremos en identificar la heterocedasticidad y, en su caso, en su posible corrección. Como se puede observar en la tabla 4 , aquí no se tiene una $\mathrm{R}^{2}$ alta con pocas $t$ significativas; sin embargo, sí existe una correlación medianamente alta entre las variables exógenas.

Tabla 4. Matriz de correlación gastos de campaña PAN

$\begin{array}{llll}\text { Variables } & \text { PROP } & \text { OPE } & \text { TV } \\ \text { PROP } & 1 & & \\ \text { OPE } & 0.830058 & 1 & \\ \text { TV } & 0.642671 & 0.667575 & 1\end{array}$

Para comenzar un análisis de heterocedasticidad, una prueba de White da como resultado un valor de $\mathrm{NR}^{2}=$ 4.8366, que, comparado con el valor de $\mathrm{X}^{2}$ al $95 \%$ de confianza, que es 16.9190, es bastante menor, por lo que se puede considerar que no existe heterocedasticidad en el modelo. Utilizando nuevamente la prueba de BreuschPagan-Godfrey, obtenemos que no existe heterocedasticidad al $99 \%$ de confianza.

Partido de la Revolución Democrática (PRD)

Para el caso del PRD, se puede observar que tanto los gastos en propaganda, como los gastos operativos son significativos, mientras que los gastos en televisión y radio no lo son. En cuanto a la eficiencia, podemos inferir que en lo que mejor se invirtió fue en gastos operativos, ya que ante un incremento porcentual en una unidad en los 
Influencia de los gastos de campaña en los resultados de las elecciones

gastos operativos, se observa un incremento en la votación de $1.2 \%$. En el caso de los gastos en propaganda ante un incremento de uno por ciento en este tipo de gastos, se dará un incremento de $0.41 \%$ en el nivel de votación captada por esta opción política.

Una $R^{2}$ ajustada con un valor de 0.83 , nos indica un que existe un buen ajuste de los datos al modelo. Continúa existiendo la posibilidad de multicolinealidad, ya que en la matriz de correlaciones existen valores altos (tabla 5). Sin embargo, como ya se mencionó, debido a la naturaleza y objetivo de este estudio no se tratará de resolver este problema.

Tabla 5. Matriz de correlación gastos de campaña $P R D$

\begin{tabular}{llll} 
Variables & PROP & OPE & TV \\
PROP & 1 & & \\
OPE & 0.871052 & 1 & \\
TV & 0.927720 & 0.939670 & 1 \\
\hline
\end{tabular}

En el caso de la heterocedasticidad, se tiene un valor $\mathrm{NR}^{2}$ en la prueba de White de 9.9227 que nos indica la existencia de homocedasticidad a un $95 \%$ de confianza. Se realizó también la prueba de Breusch-Pagan-Godfrey y ambas pruebas arrojan evidencia que nos permite asumir que no existe heterocedasticidad en nuestro modelo.

Partido Revolucionario Institucional (PRI)

Para el Partido Revolucionario Institucional, se observa que las tres variables, gastos en propaganda, gastos operativos y gastos en radio y televisión son significativas. En cuanto a la eficiencia en el PRI, podemos inferir que en lo que mejor se invirtió fue en gastos en radio y televisión, ya que ante un incremento porcentual en una unidad en los gastos en este rubro, se observa un incremento en la vota- 
ción por este partido de $0.43 \%$; en el caso de los gastos en propaganda ante un incremento de uno por ciento en este tipo de gastos, se dio un incremento de $0.32 \%$ en el nivel de votación captada por este partido; y en el caso de los gastos operativos, ante un incremento de uno por ciento en ellos se observa un incremento en $0.20 \%$ en los votos.

Una $\mathrm{R}^{2}$ ajustada con un valor de 0.94 , nos indica que existe un buen ajuste de los datos al modelo. Existe también en este caso la posibilidad de problemas de multicolinealidad, ya que la matriz de correlaciones presenta valores cercanos a 0.9. Sin embargo como en los otros casos, únicamente se tendrá conciencia de las implicaciones derivadas de ello, pero no se intentará resolver el problema, pues la totalidad de nuestras observaciones es sólo de 32. Esta situación nos impide incrementar el tamaño de nuestra muestra, acción que se considera la mejor forma de resolver problemas de este tipo.

Tabla 6. Matriz de correlación gastos de campaña PRI

$\begin{array}{llll}\text { Variables } & \text { PROP } & \text { OPE } & \text { TV } \\ \text { PROP } & 1 & & \\ \text { OPE } & 0.863149 & 1 & \\ \text { TV } & 0.868232 & 0.879713 & 1\end{array}$

En el caso de la heterocedasticidad, se tiene un valor $\mathrm{NR}^{2}$ en la prueba de White de 10.1969, lo que nos indica la existencia de homocedasticidad al 95\% de confianza. Nuevamente se calculó la prueba de Breusch-Pagan-Godfrey. En esta ocasión se encontró que existe homocedasticidad a $99 \%$ de confianza.

Partido del Trabajo (PT)

Del modelo estimado, se observa que la variable que para el PT tuvo más peso fueron los gastos en radio y televisión, 
Influencia de los gastos de campaña en los resultados de las elecciones

ya que ante un incremento porcentual de una unidad en estos gastos, se tiene un incremento de $0.54 \%$ en la votación recibida por este partido; además de contar con un valor $t$ que hace al parámetro significativo a $95 \%$ de confianza. Para el caso de los gastos en propaganda, se tiene que ante un incremento porcentual de uno en propaganda, se tiene un aumento de la votación en $0.38 \%$; con una $t$ que da significación a los parámetros a $90 \%$ de confianza. Finalmente, los gastos operativos no son significativos.

Se tiene una $\mathrm{R}^{2}$ ajustada con un valor de 0.5370 , lo que indica un no muy buen ajuste del modelo en su conjunto. En la Tabla 7 se puede observar que, a diferencia de los otros partidos, los valores de la matriz de correlación no son tan altos, por lo que la probabilidad de estar en presencia de multicolinealidad es menor.

Tabla 7. Matriz de correlación gastos de campaña $P T$

\begin{tabular}{llll} 
Variables & PROP & OPE & TV \\
PROP & 1 & & \\
OPE & 0.513791 & 1 & \\
TV & 0.674749 & 0.634371 & 1 \\
\hline
\end{tabular}

$\mathrm{Al}$ igual que en los otros casos, se realizó la prueba de heterocedasticidad de White y se encontró un valor $\mathrm{NR}^{2}$ de 9.6592, lo que nos indica homocedasticidad a 95\% de confianza. La prueba de Breusch-Pagan-Godfrey indica homocedasticidad a $99 \%$ de confianza, por lo que se puede considerar que el modelo del Partido del Trabajo tampoco presenta este problema.

Partido Verde Ecologista de México (PVEM)

Cabe hacer la observación de que como no se contaba con información principalmente en los rubros de gastos operativos y en propaganda para todos los estados, la estimación del 
PVEM se realizó únicamente con los 15 estados que contaban con los tres rubros. Pareciera que el PVEM no gastó en estos rubros, primero por sus convicciones partidistas ligadas a la ecología, y segundo porque en esos lugares no contaba con suficientes militantes que lo apoyaran en la campaña, por lo que decidió utilizar únicamente la televisión y el radio.

En el modelo estimado se observa que la variable que influye más en la votación por el PVEM, es precisamente la correspondiente a los gastos en radio y televisión, ya que ante un incremento porcentual de una unidad en estos gastos, se tiene un incremento de $0.99 \%$ en la votación recibida por este partido; además, cuenta con un valor $t$ que hace al parámetro significativo a $99 \%$ de confianza.

Tanto los gastos en propaganda como los gastos operativos no se consideran significativos pues los valores críticos de las pruebas t son muy bajos, lo que incrementa la probabilidad de que los parámetros de estas dos variables sean cero y no influyan en la variable endógena. Se tiene una $\mathrm{R}^{2}$ ajustada con un valor de 0.8141 , lo que indica un buen ajuste del modelo en su conjunto, además de un valor de la prueba $\mathrm{F}$ con una probabilidad alta, lo que confirma que la menos una variable del modelo es significativa.

Al igual que el Partido del Trabajo, el Partido Verde Ecologista de México presenta valores de la matriz de correlación que no son tan altos como en los casos de los otros partidos políticos, por lo que se puede considerar que existen menos probabilidades de multicolinealidad.

Tabla 8. Matriz de correlación gastos de campaña PVEM

\begin{tabular}{llll} 
Variables & PROP & OPE & TV \\
PROP & 1 & & \\
OPE & 0.093907 & 1 & \\
TV & 0.561422 & 0.406631 & 1 \\
\hline
\end{tabular}


Influencia de los gastos de campaña en los resultados de las elecciones

Aplicando la prueba de White, se tiene un valor de $\mathrm{NR}^{2}=$ 9.6614, que también es menor al valor de $\mathrm{X}^{2}$ de tablas a $95 \%$ de confianza, por lo que se puede considerar que no existe heterocedasticidad en el modelo. La prueba de BreuschPagan-Godfrey indica que existe homocedasticidad a $99 \%$ de confianza.

\section{Reflexiones finales}

Es importante aclarar que este trabajo parte de la hipótesis de que las campañas políticas influyen los resultados de las elecciones. Existen otros factores que pueden condicionar o afectar de alguna forma la decisión de votar por determinada alternativa política. Sin embargo, muchos de esos otros factores son, al menos en forma indirecta, afectados por la disponibilidad de recursos para llevar a cabo las campañas. Este estudio analiza la influencia de los gastos de campaña en los resultados de las elecciones federales de 1997.

Como se puede observar, la influencia que parecieran tener los gastos de campaña en la votación obtenida no es muy fuerte. Una de las posibles razones de ello es que únicamente se incluyeron variables de gastos de campaña en el modelo y tal vez existen efectos de interacción entre las variables de gastos y otras variables independientes. Sin embargo, como el objetivo de este trabajo es precisamente mostrar la importancia de estos gastos, se consideró pertinente y necesario no incluir otras variables explicativas importantes como población, educación o actividad económica preponderante.

Incluso en este caso extremo, en el que las variables relativas a gastos de campaña estén captando parte de la variación explicada por otras variables, los coeficientes obtenidos son muy pequeños. Esta situación nos hace pensar que la incidencia de los gastos de campaña —o al menos de 
los gastos que los partidos informan de manera formal-, en los resultados de las elecciones, es relativamente débil.

Por otra parte, los gastos de campaña no necesariamente son aplicados en su totalidad a herramientas y técnicas de mercadotecnia política. Muchos políticos los pueden utilizar con fines distintos a los meramente electorales; por lo tanto, esto puede variar considerablemente según cada campaña política, cada partido o incluso cada candidato.

Observando los resultados de los primeros dos modelos, es claro que existe una influencia relativamente baja entre los gastos de campaña y la votación total emitida. Por lo tanto, no se puede considerar que las grandes inversiones de fondos públicos en las campañas electorales ayuden de una forma significativa a combatir el abstencionismo y a aumentar los porcentajes de participación ciudadana.

$\mathrm{Al}$ analizar el primer modelo, como ya lo expresamos, la constante es negativa, lo que en la práctica es imposible, ya que no se pueden emitir votos negativos; pero puede indicarnos que, sin gastos de campaña, los posibles votos emitidos serían cero; es decir, nadie estaría dispuesto a votar en caso de no existir grandes cantidades invertidas en las campañas electorales. Ello tal vez debido a que la gente no tendría suficiente información sobre las elecciones y los diferentes candidatos en la contienda.

En este primer modelo la variable que más influye en la votación de los cinco partidos en su conjunto, es la propaganda en una relación significativa de 11\%. Después los gastos operativos, con una relación significativa de 6\%; y finalmente los gastos en televisión y radio, los cuales además de tener un peso muy bajo en su asociación con el modelo, tienen una prueba t no significativa, lo que nos dice que probablemente no existe relación causal entre estos gastos y la votación total emitida.

En el segundo modelo de abstencionismo, en donde se sumaron los gastos de campaña para convertirlos en una 
Influencia de los gastos de campaña en los resultados de las elecciones

sola variable, los gastos de campaña inciden de forma significativa en los resultados de la votación total, con un coeficiente de 0.063 . La variable exógena es significativa y la $\mathrm{R}^{2}$ tiene una magnitud de 0.96 , por lo que el ajuste de los datos al modelo es bueno.

Como podemos observar, en este segundo modelo de abstencionismo, si bien eliminamos el problema de multicolinealidad, es imposible saber si cierto tipo de gasto tiene mayor influencia en la votación total emitida, pues todos los gastos están incluidos en una sola variable independiente.

Por otro lado, en el caso de la eficiencia en los gastos de los distintos partidos, para Acción Nacional los gastos en propaganda y los gastos en radio y televisión le representan una mayor cantidad de votos por unidad de gasto. De hecho, un cambio en $0.52 \%$ en la votación obtenida por cada incremento de $1 \%$ en el gasto en propaganda, y un aumento de $0.55 \%$ en los votos por cada incremento de $1 \%$ en los gastos utilizados para televisión y radio.

Para el caso del PRD, se puede observar que tanto los gastos en propaganda como los gastos operativos son significativos, mientras que los gastos en televisión y radio no lo son. En cuanto a la eficiencia, en lo que mejor invirtió el PRD fue en gastos operativos, ya que ante un incremento porcentual en una unidad en los gastos operativos, se observa un incremento en la votación de $1.2 \%$. En el caso de los gastos en propaganda, ante un incremento de uno por ciento en este tipo de gastos se dio un incremento de $0.41 \%$ en el nivel de votación captada por esta opción política.

En el caso del PRI, se observa que las tres variables exógenas, gastos en propaganda, gastos operativos y gastos en radio y televisión son significativas. En cuanto a la eficiencia en el PRI, podemos inferir que en lo que mejor se invirtió fue en gastos en radio y televisión, ya que ante un incremento porcentual en una unidad en los gastos en este rubro, se 
observa un incremento en la votación por este partido de $0.43 \%$. Para el caso de los gastos en propaganda ante un incremento de uno por ciento en este tipo de gastos, se dará un incremento de $0.32 \%$ en el nivel de votación captada por este partido. Finalmente, en el caso de los gastos operativos, ante un incremento de uno por ciento en ellos se observa un incremento en $0.20 \%$ en los votos.

Del modelo estimado para el PT, se observa que la variable que más peso tuvo en su votación es gastos en radio y televisión, ya que ante un incremento porcentual de una unidad en estos gastos, se tiene un incremento de $0.54 \%$ en la votación recibida por este partido. Para el caso de los gastos en propaganda, se tiene que ante un incremento porcentual de uno en propaganda, se tiene un aumento de la votación en $0.38 \%$.

En el modelo estimado para el PVEM se observa que la variable que más influye es gastos en radio y televisión; ya que ante un incremento porcentual de una unidad en estos gastos, se tiene un incremento de $0.99 \%$ en la votación recibida por este partido. Los gastos en propaganda y los gastos operativos no resultaron significativos. Sin embargo, ello puede ser, al meno en parte, el resultado de la manipulación de los datos que se tuvo que realizar con la finalidad de correr el modelo. Como se mencionó antes, no se contaba con información de este partido para todos los estados, por lo que se decidió reducir el número de observaciones a 15 entidades federativas.

Por último, como se puede observar, las distintas variables utilizadas en los modelos influyen de formas muy diferentes en los distintos partidos políticos. Lo que para un partido puede ser muy importante, para otro tiene menos relevancia en la totalidad de sus esfuerzos por conseguir votos. Los resultados de este estudio muestran lo importante que es estudiar la eficiencia del gasto en campañas electorales. Una línea de investigación importante sería continuar 
Influencia de los gastos de campaña en los resultados de las elecciones

con este tipo de estudios en un intervalo de tiempo amplio, lo cual nos llevaría a obtener conclusiones valiosas acerca de la eficiencia de cada rubro de gasto para cada uno de los partidos políticos existentes.

De la misma forma se podría analizar la eficacia del financiamiento público para incentivar el voto ciudadano, y por tanto reducir el abstencionismo. La utilización de financiamiento público no es o no debería ser únicamente una forma de equidad entre las diferentes fuerzas políticas, sino también una inversión social que tenga como resultado una mayor participación ciudadana en los comicios. La reducción del abstencionismo tiene el potencial de lograr elecciones con niveles altos de legitimidad, los cuales son muy importantes para países que, como México, están renovando o fortaleciendo sus instituciones democráticas.

Bibliografía

Alker, H. R., "Statistics and Politics: The Need for Causal Data Analysis", en S. M. Lipset, Politics and the Social Sciences, Oxford University Press, 1970.

Arbesú, L., Mercadotecnia política, México: IFE (col. Cuadernos de orientación metodológica, núm. 2), 1998.

Barranco, F. Javier, Técnicas de marketing político, México, REI, 1994.

Fisher, Laura, Mercadotecnia, México, McGraw-Hill, 1993.

Gujarati, D., Econometría, Colombia, McGraw-Hill, 1997.

Intriligator, Michael D., Modelos econométricos, técnicas y aplicaciones, México, FCE, 1990.

Kennedy, Peter, Introducción a la econometría, México, FCE, 1996.

Kirmani A., Wright P., "Money talks perceived advertising expense and expected product quality", en Journal of Consumer Research, vol. 16, diciembre, 1989, pp. 344353.

Kotler, Philip, Marketing for nonprofit organizations, Estados Unidos, Prentice-Hall, 1982. 
Luque, Teodoro, Marketing político. Un análisis de intercambio político, Barcelona, Ariel, 1996.

Maarek, P., Marketing político y comunicación. Claves para una buena información política, Buenos Aires, Paidós Comunicación, 1997.

Maddala, G. S., Introducción a la econometría, México, Prentice-Hall, 1996.

Martin, L., Marketing político. Arte y ciencia de la persuasión en democracia, Buenos Aires: Paidós, 2002.

Martínez Silva, Mario, Manual de campaña: Teoría y práctica de la persuasión electoral, México, Colegio Nacional de Ciencias Políticas y Administración Pública, 1998.

Naghi Namakforoosh, Mohammad, Mercadotecnia electoral, tácticas y estrategias para el éxito político, México, Limusa, 1984.

O'Cass, Aron, "Political marketing an investigation of the political marketing concept and political market orientation in Australian politics", en European Journal of Marketing, vol. 35, núms. 9 y I0, 200I, Pp. I003-1025.

Payne, J. L., "Elections and government spending", en Public Choice, vol. 70, I99I, pp. 7I-82.

Stanton, William et al., Fundamentos de marketing, México, McGraw-Hill, 1996. 